\title{
How About Effect of Lithium Carbonate on Preventive Switch Induced by Antidepressants in Patients With Depressive Episode? Chinese Data Analysis
}

\section{Wangqiang Lv ( $\sim$ lvwangqiangjh@163.com )}

Jinhua Second Hospital

\section{Tao Hejian}

Zhejiang Chinese Medical University

\section{Sun Fengli}

Zhejiang Province mental Health Center

Jin Weidong

Zhejiang College of Traditional Chinese Medicine: Zhejiang Chinese Medical University

\section{Primary research}

Keywords: Lithium carbonate, Depressive episode, Switch rate

Posted Date: January 15th, 2021

DOl: https://doi.org/10.21203/rs.3.rs-143728/v1

License: (c) (1) This work is licensed under a Creative Commons Attribution 4.0 International License.

Read Full License 


\section{Abstract}

Background: Although mania or hypomania was defined as indispensable for bipolar disorder, depressive episodes are more common and impairing, with proven response to treatments.So the prevention switch was a important affair in clinical psychiatry

Methods: We searched CBM, CNKI, WANFANG and CSSCI in Chinese to find literature from July 12000 to July 312020 related to the study in model of "comparison of switch rate between combination treatment of lithium and antidepressant and monotherapy of antidepressant in patients with depressive episode", among which results such as comments, letters, reviews and case reports were excluded. The rate of switch between groups was synthesized and discussed.

Result: A total of 695 subjects were included in 9 studies. Random effect model is used to account for the data by Revman 5.2. The results showed that the switch rate of lithium carbonate was $8.28 \%$ (29/350),switch rate of antidepressant was $25.29 \%(87 / 344)$, which was very different in switch rate(OR=0.25, 95\% Cl: $0.16-0.39)$ and also indicated that lithium reduced switch rate was $67.25 \%$ (25.29\%-8.28\%/25.29\%). In bipolar depression group, lithium reduced switch rate was $68.11 \%$ (25.84\%-8.24\%/25.84\%). In depression group, lithium reduced switch rate was $67.34 \%$ (25.29\%-8.26\%/25.29\%). In group of patients treated by SSRI, lithium reduced switch rate was $60.3 \%$ (29.85\%-11.85\%/29.85\%).In group of patients treated by TCA, lithium reduced switch rate was $73.14 \%$ $(22.28 \%-6.01 \% / 22.28 \%)$.

Conclusion: As typical mood stabilizer, lithium carbonate can reduced switch rate related to antidepressant in patients with depressive episode.

\section{Background}

As a classic mood stabilizer, lithium carbonate can be used in depressive episode for the following reasons.First,lithium carbonate has a obvious action of anti-suicide[1,2], because suicide is a emergency in psychiatric clinic,especially in adolescent bipolar disorder,which always is with trait of suicide[2].Second,lithium has anti-depressant role.Taking into account good adherence to drug treatment, in clinical practice settings, long-term lithium salts seem to have a preventive effect on depressive symptoms[3]. Third,lithium can strengthen the role of antidepressant in treatment for depression.This point views was proven in $S T A R^{\star} D$, although more participants discontinued lithium due to adverse effects than discontinued others[4].Forth, lithium had a same anti-depressant role as lamotrigine in bipolar depression,late was called "mood stabilizer for depression" and they all are recommended for bipolar depression in treatment guideline of bipolar disorder[5,6].So many studies results are the reasons of lithium in treatment for depressive episode.

Another important reason is that lithium carbonate can prevent switch induced antidepressant,which is a special phenomenon in the clinical process of psychiatry. This is neither a side effect of the 
switch included age of onset, personality characteristics, family history, type of antidepressants, type of depression,combination without mood stabilizers,previous history, severe suicide attempts, amphetamine use and some combination of pharmacological treatments. During the current depressive episode, the identified risk factors were any possible mood elevation, multiple mania-associated symptoms with at least moderate severity, and comorbid panic attacks.[8,9].In general,adjunctive antidepressants are associated with reduced symptoms of acute bipolar depression, but the magnitude of benefit is small because they do not increase clinical response or remission rates. However, these medications should be used only in the short term because prolonged use is associated with an increased risk of treatmentemergent mania or hypomania[10].It had been found switch rate of bipolar depression had very higher than that in "nuipolar"depression[11].Patients with bipolar depression present very heterogeneous responses to the use of ADs. Some improve significantly, while others, especially those with concomitant manic symptoms, have had previous episodes of treatment-emergent mania or are rapid cyclers, exhibit manic switches or cycle acceleration[12]. The one of methods in avoidance switch was use of mood stabilizer,especially lithium carbonate[3,10,11,12].

Mood stabilizer can reduce the risk of developing antidepressant-induced manic form states in acute treatment of bipolar I depressed patients[13].In 2007,we found that classic mood stabilizer, lithium carbonate can reduced $62 \%$ switch rate associated with antidepressant[14]. The following study continuously indicated that lithium carbonate reduced $66.4 \%$ switch rate associated with antidepressant by meta-analysis[15].These results show sue of lithium carbonate can reduce the switch in depression,which not all are bipolar depression in China.In recent years,the research has increased about comparison between combination treatment of lithium and antidepressant and monotherapy of antidepressant in patients with depressive episode,in which the switch rate was noticed. The further study is necessary comparing switch rate between combination treatment of lithium and antidepressant and monotherapy of antidepressant in patients with depressive episode by meta-analysis and assessment of prevention about switch rate of lithium in patients with depressive episode.

\section{Methods}

\section{Literature retrieval methods:}

1.1 This study was performed according to the recommendations of the Moose [16]. Two reviewers independently searched the database. The database includes just Chinese databases; Searches were applied to the following Chinese electronic databases: Chinese Biomedical Database (CBM), China National Knowledge Infrastructure (CNKI), WANFANG and Chinese Social Sciences Citation Index (VIP) databases .

1.2 Search key words: Depression (bipolar depression,depressive episode); lithium carbonate;

1.3 The search strategy:The search strategy was based on combinations. We also modified the terms arrordina th the different datahaces Tn retrieved all articles, we first search papers by "lithium carbonate Loading [MathJax]/jax/output/CommonHTML/fonts/TeX/fontdata.js 
and depression", And then further screen the papers related to lithium carbonate and depression.Last query was updated on late 1 January 2000 to 31 July 2020. References of retrieved articles were crosssearched to identify any studies missed by the electronic search strategies.

\subsection{Inclusion and Exclusion Criteria}

The two researchers reviewed the initial retrieved publications independently. The discrepancy was resolved through discussion by all reviewers. Studies that met the following general criteria were "comparison between combination treatment of lithium and antidepressant and monotherapy of antidepressant in patients with depressive episode". (1) detection rate of mania or hypomania was reported during study; or (2) detection rate of psychopathology of very irritable or very compulsive to have to change therapeutic plan or treated as drop off cases or (3)cases changed mixed episode from bipolar depression or (4) YMRS or BRMS was higher 11 score. However, articles had incomplete or unidentified data were excluded, as well as abstracts, reviews, case reports, letters and duplicate publications.

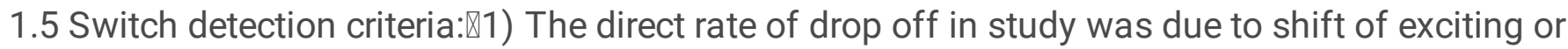
hypomania or mania.(2) The detection rate of or cases of switch was reported directly in study.(3) The rate of changing therapeutic plan was due to psychopathology of mania or hypomnia or very irritable or very compulsive.(4) The the case numbers of study patients with higher 11 score of YMRS or FMRS . (5)The cases were reported in study,which became mixed episode from bipolar depression.

1.6 Two psychiatrists reviewed each included article independently, using the 11-item checklist that was recommended by the Agency for Healthcare Research and Quality (AHRQ) [16]. An item would be scored ' 0 ' if it was answered 'NO' or 'UNCLEAR' whereas ' 1 ' will be given to the answer 'YES'. Article quality was assessed as follows: low quality $=0-3$; moderate quality $=4-7$; high quality $=8-11$. Differences in article quality were discussed to reach an agreeable final score. The following information was extracted: first author, publication time, the sample size, study population, assessment tools, and the number of people who had anxiety and depression,yes or no comparison.

1.7 Statistic analysis:All statistical analyses were performed using Statistical Analysis System software (Revman 5.2), and the $P$ value for the overall effect $<0.05$ with two-tailed was considered statistically significant. The heterogeneity of all involved studies was assessed by $\mathrm{I}^{2}$. When it was lower than $50 \%$, the studies with an acceptable heterogeneity were considered, and then the fixed-effects model with MantelHaenszel method was used; otherwise, a random effect model with the Der Simonian and Laird (DL) method was adopted. The combined odds ratio (OR) were initially estimated using Forrest plots raphically. For each trial, the $\mathrm{OR}$ was estimated from the original article. If not available, we looked at the total numbers of events and the numbers of patients at risk in each group to determine the OR estimate.

1.8 Assessment of publication bias was investigated for each of the pooled study groups mainly by the Egger's linear regression test. As supplement approach, the Begg's rank correlation also was applied to assess the potential publication bias, when $\mathrm{P}<0.05$ was considered that there was no publication bias in 


\section{Results}

\section{Study Characteristic}

Nine comparison studies, with 695 subjects, met the inclusion criteria and were included for the final meta-analysis[17,18,19,20,21,22,23,24,25]. The 695 subjects consist of 351 cases in experimental group and 344 cases in control group. The sample size of the studies ranged from 44 to 105. The years of nine studies are from 2001 to 2015 . 117 cases switch from depression to mania in total.

\section{Comparison of switch rate between experimental group and control group.}

A total of 695 subjects were included in 9 studies. Random effect model is used to account for the data by Revman 5.2. The results showed that the switch rate of lithium carbonate was $8.28 \%(29 / 350)$,switch rate of antidepressant was $25.29 \%$ (87/344), which was very different in switch rate(OR=0.25,95\% Cl: $0.16-0.39)$.The switch rate in experimental group was significantly lower than in that in control group $(\mathrm{Z}=5.9, \mathrm{P}<0.00001)$. And it also indicated that lithium reduced switch rate was $67.25 \%($ $25.29 \%-8.28 \% / 25.29 \%)$.See figure2.

The funnel plot analysis of study about switch rate show a gap,which indicate there maybe a bias of publication.see figure3.But the Eggerspublicationbiastest $(P=0.16)$ and Beggs publication bias test $(P=0.62)$ all show the there not were publication bias.see figure4 and figure5.

\section{Subgroup comparison of switch rate}

\subsection{Subgroup comparison of switch rate according to type of depression.}

A total of 186 bipolar subjects were included in 3 studies. Random effect model is used to account for the data by Revman 5.2. The results showed that the switch rate of lithium carbonate group was $8.25 \%$ (8/97),switch rate of antidepressant group was $25.84 \%$ (23/89) in bipolar patients, which was very different in switch rate(OR=0.24,95\% $\mathrm{Cl}$ : $0.10-0.58)$. The switch rate in experimental group was significantly lower than in that in control group $(Z=3.19, P=0.001)$. In bipolar depression group, lithium reduced switch rate was $68.11 \%(25.84 \%-8.24 \% / 25.84 \%)$. See figure6.

A total of 509 unibipolar subjects were included in 6 studies. Random effect model is used to account for the data by Revman 5.2. The results showed that the switch rate of lithium carbonate group was $8.26 \%$ (21/254),switch rate of antidepressant group was $25.09 \%$ (64/255) in unibipolar patients, which was very different in switch rate(OR=0.25,95\% $\mathrm{Cl}$ : $0.15-0.43)$. The switch rate in experimental group was significantly lower than in that in control group $(Z=4.96, P<0.0001)$. In depression group, lithium reduced switch rate was $67.07 \%$ (25.09\%-8.26\%/25.09\%). See figure6.

\subsection{Subgroup comparison of switch rate according to type of antidepressant.}

A total of 426 subjects were included in 5 studies according to TCA treatment study. Random effect Loading [MathJax]/jax/output/CommonHTML/fonts/TeX/fontdata.js e results showed that the switch rate of lithium 
carbonate group was $6.01 \%(13 / 216)$,switch rate of antidepressant group was $22.38 \%(47 / 210)$, which was very different in switch rate(OR=0.20,95\% $\mathrm{Cl}$ : $0.10-0.40)$. The switch rate in experimental group was significantly lower than that in control group $(Z=4.66, P<0.00001)$. Lithium reduced switch rate was $73.14 \%(22.38 \%-6.01 \% / 22.38 \%)$ in patients treated with TCA. See figure7.

A total of 269 subjects were included in 4 studies according to SSRI treatment study. Random effect model is used to account for the data by Revman 5.2. The results showed that the switch rate of lithium carbonate group was $11.85 \%(16 / 135)$,switch rate of antidepressant group was $29.85 \%$ (40/134), which was very different in switch rate(OR=0.30,95\% $\mathrm{Cl}$ : $0.16-0.58)$. The switch rate in experimental group was significantly lower than that in control group $(Z=3.62, P=0.0003)$. Lithium reduced switch rate was $60.30 \%$ (29.85\%-11.85\%/29.85\%). See figure7.

\section{Discussion}

Lithium has been an intriguing treatment option in psychiatry for over a century. While seemingly just a simple elemental compound, it has powerful treatment effects for both depression and bipolar disorder. Lithium is still one of few drugs that have been proven to reduce the risk of suicidality and prevention for depressive symptoms, and it may have utility in illnesses beyond affective disorders in recent years[2,3]. Practically, as a primary agent or as an adjunct, lithium continues to claim a rightful place in the treatment for adjunctive for depression[17,18,19,20,21,22]. In China,many studies have proven that lithium carbonate combination with antidepressant is more effective than that monotherapy by antidepressant in management of depression,especially in irritability,compulsive behavior[17-22].

The certain number of depressive patients switch to mania or exciting status during treatment by antidepressant, of which could be diagnosed by criteria of bipolar disorder in DSM-5[26 ].But this is not successful therapeutic plan for patient duo to switch,because it induce the mania ahead[7].So avoiding switch to mania is important part of therapeutic plan,whereas the patients is unipolar or bipolar depression.In general, four status indicate the patients come into switch.First is mania or hypomania.Second is primary rapid cycle become acceleration, Third is obvious and serious behavior of irritability,compulsive,agitated behavior to have to change manege methods during treatment,Fourth is antidepressant induced chronic irritability(AICD)[8]. These all indicate the patients switch to mania,which suggest that the therapeutic plan should be changed.

To recognize the risk of switch was one of prevention methods before treatment[8,9].And therapeutic drug was also very important. In Cox proportional hazard analyses, both antidepressant monotherapy and polytherapy exhibited higher risk of manic switch than their alternatives (antidepressant monotherapy vs. SGA monotherapy, hazard ratio [HR]=2.87 [95\% Cl: 1.10-7.49][27]. So establishing of correct management program is necessary. The add on mood stabilizer,such as lithium carbonate maybe one important methods in treatment of patients with depressive episode if possible.

This meta-analysis found switch rate of lithium carbonate group was $8.26 \%(21 / 254)$, switch rate of Loading [MathJax]/jax/output/CommonHTML/fonts/TeX/fontdata.js patients, which was very different in switch 
rate(OR=0.25,95\% Cl: $0.15-0.43)$. The switch rate in experimental group was significantly lower than in that in control group $(Z=4.96, P<0.0001)$. But the funnel plot analysis of study about switch rate show asymmetric due to a gap,which indicate there maybe a bias of publication. Whereas the Egger spublicationbiastest $(P=0.16)$ and Beggs publication bias test $(P=0.62)$ all show the there not were publication bias. This suggest lithium carbonate do decrease the switch rate induced by antidepressant during treatment for patients with episode of depression.

It was known to all that different depression and different antidepressant were accompanied different switch rate[7,8,9].So the subgroup analysis was made. The result also show lithium carbonate can decreased the switch rate both in patients with unipolar depression and bipolar depression, although bipolar disorder have higher switch rate than that in unipolar disorder[28,29]. The result also show lithium carbonate can decreased the switch rate both in patients treated with TCA and SSRI, although switch rate related to TCA was higher than that related to SSRI[29,30]. These all means that lithium carbonate reduce switch rate regardless of the type of antidepressant and the type of depressive episode.

The switch-inducing potential of antidepressants is unclear, although tricyclic antidepressants, which confer higher risk of switching than other classes of antidepressants, with higher NE function in CNS, are a possible exception. Converging evidence suggests that certain pharmacologic and nonpharmacologic interventions with very different mechanisms of action, such as sleep deprivation, exogenous corticosteroids, and dopaminergic agonists, can trigger mood episode switches in patients with bipolar disorder or soft bipolar disorder[31]. But lithium carbonate may displace $\mathrm{Na}+$ from the allosteric $\mathrm{Na}+-$ binding sites in neurotransmitter transporters and G-protein coupled receptors (GPCRs), which can stabilize the unsettle of mood[32].

This study had several limitations. Firstly, the sample size of this meta-analysis was relatively small. Only 9 studies and 695 subjects were involved. Secondly, collecting data style may influence the result of investigation, for example,different criteria of switch can get different detection rate of switch. so it was very import to establish a diagnostic criteria for switch associated with antidepressant.Thirdly, not all the studies had blind observation. .These factors are partly responsible for the source of pool rate of switch associated with antidepressant, also affect us to see the real significance and risk of switch.

\section{Conclusion}

As typical mood stabilizer, lithium carbonate can reduced switch rate related to antidepressant in patients with depressive episode. In this study, lithium carbonate reduce $67.25 \%$ switch rate regardless of the type of antidepressant and the type of depressive episode.But the ability of lithium carbonate in reducing switch is not the reason of use about antidepressant in bipolar depression. The 3 principles should be taken during treatment for bipolar depression,which are no first selection of antidepressant,no monotherapy by antidepressant,no combination of antidepressants. 
MEDLINE=The National Library of Medicine

EMBASE=Excerpta Medical Database

$\mathrm{CBM}=$ Chinese Biomedical Database

CNKI=China Nationa IKnowledge Infrastructure

$\mathrm{CSSCl}=$ Chinese Social Sciences Citation Index (VIP)

YMRS=Yung Manic Rating Scale

BRMS=Beck-Rafaelsdn Mania Rating Scale

\section{Declarations}

\section{Ethics approval and consent to participate}

Not Available

\section{Consent to publication}

All authors agree to publish our paper and no conflict in any interests.

\section{Availability of data and material.}

See Table1

\section{Competing interests}

There were not any financial and non-financial competing interests.

\section{Funding}

Not Available

\section{Authou's contribution}

Our authors have different contributions to this article. Dr LWQ participated in collection of data and the writing of the article, LWQ and Dr FWX,SFL assessed the quality of researched papers. Dr THJ complete most statistic analysis.All authors reviewed all researched paper. Prof JWD participated in the design , statistical processing and the final revision of the article.

\section{Acknowledgment}

We thank Mr Xv Songquan give us study idea and Mr Wang Zhiqiang help us in literature retrieval. We Loading [MathJax]/jax/output/CommonHTML/fonts/TeX/fontdata.js of Ma Yongchun in final revision of the article. 


\section{References}

1. Ramadhan Oruch, Mahmoud A Elderbi , Hassan A Khattab , lan F Pryme, Anders Lund.Lithium: a review of pharmacology, clinical uses, and toxicity,Eur J Pharmacol. 2014, 5;740:464-73.doi: 10.1016/j.ejphar.2014.06.042.

2. B Grant , J A Salpekar .Using Lithium in Children and Adolescents with Bipolar Disorder: Efficacy, Tolerability, and Practical Considerations,Paediatr Drugs. 2018 ;20(4):303-314.doi: 10.1007/s40272018-0289-x.

3. Ana González-Pinto , Purificación López-Peña , Cristina Bermúdez-Ampudia , Eduard Vieta , Mónica Martinez-Cengotitabengoa .Can lithium salts prevent depressive episodes in the real world?Eur Neuropsychopharmacol. 2018 Dec;28(12):1351-1359.doi: 10.1016/j.euroneuro.2018.09.008.

4. Rush, J.A.,Warden, D.,Wisniewski, S.R.,Fava, M.,Trivedi, M.H.,Gaynes, B.N.,Nierenberg, A.A.STAR*D: Revising conventional wisdom.CNS Drugs, 2009,23 (8) : 627-647.

https://doi.org/10.2165/00023210-200923080-00001

5. Jin Rui,Chen Fengpei,Zhu Jianfeng, Gao Zhihan, Shen Ying, Chen Zhengxin, Ren Zhibin,Ma Yongchun and Jin Weidong. Lthium carbonate and Lamotrigine for Treatment of Bipolar Depression:Which is Better? Results From Chinese Data in Meta-analysis. EC Psychology and Psychiatry, 2018,7(12):992-1002

6. Lakshmi N Yatham, Sidney H Kennedy, Sagar V Parikh, Ayal Schaffer, David J Bond, Benicio N Frey, Verinder Sharma, Benjamin I Goldstein, Soham Rej, Serge Beaulieu, Martin Alda, Glenda MacQueen, Roumen V Milev, Arun Ravindran, Claire O'Donovan, Diane McIntosh, Raymond W Lam, Gustavo Vazquez, Flavio Kapczinski, Roger S McIntyre, Jan Kozicky, Shigenobu Kanba, Beny Lafer, Trisha Suppes, Joseph R Calabrese, Eduard Vieta, Gin Malhi, Robert M Post, Michael Berk Canadian Network for Mood and Anxiety Treatments (CANMAT) and International Society for Bipolar Disorders (ISBD) 2018 guidelines for the management of patients with bipolar disorder.Bipolar Disord. 2018 Mar;20(2):97-170.doi: 10.1111/bdi.12609.

7. Jin Weidong, Tang Jianliang. Bipolar Mood Disorder,People`s Millitary Medical Press,First edtion,2011

8. Gao Zhihan, Jin Weidong.Antidepressants and switch.Sichuan Mental Health.2015,28(1):1416,doi:10.11886/j.issn.1007-3256.2015.01.005

9. Tomihisa Niitsu , Chiara Fabbri , Alessandro Serretti .Predictors of switch from depression to mania in bipolar disorder.J Psychiatr Res,2015;66-67:45-53. doi: 10.1016/j.jpsychires.2015.04.014.

10. Alexander McGirr , Paul A Vöhringer, S Nassir Ghaemi , Raymond W Lam , Lakshmi N Yatham. Safety and efficacy of adjunctive second-generation antidepressant therapy with a mood stabiliser or an atypical antipsychotic in acute bipolar depression: a systematic review and metaanalysis of randomised placebo-controlled trials. Lancet Psychiatry. 2016;3(12):1138-1146.doi: 10.1016/S2215-0366(16)30264-4. 
11. Wen Lu, Li Bingling, Ma Yongchun, Chen Jiong and Jin Weidong. Prospective observation of switching rate of antidepressants in management of depressive episode in 3 months. Sichuan Mental Health, 2015,28(1):1-3. doi:10.11886/j.issn.1007-3256.2015.01.001

12. Elie Cheniaux , Antonio E Nardi.Evaluating the efficacy and safety of antidepressants in patients with bipolar disorder.Expert Opin Drug Saf. 2019 ;18(10):893-913. doi: 10.1080/14740338.2019.1651291.

13. Bottlender R, Rudolf D, Strauss A, Möller HJ. Mood-stabilisers reduce the risk of developing antidepressant-inducedmaniform states in acute treatment of bipolar I depressed patients.J Affect Disord. 2001 ;63(1-3):79-83. doi: 10.1016/s0165-0327(00)00172-5.

14. Jin Xueguang, Jin Weidong.Lithium carbnate reduce the switc rate induced by antidepressant:metaananlysis of domestic data.Arch of Shanghai Psychiatry, 2007,19(5):313-

314.doi:10.3969/jissn.1002-0829.2007.05.018

15. Yao Lihua, Wang Xiaoping, Wang Huiling, Wang gaohua and liu Zhongchun.Meta-analysis of ruduction of turning manic rate by lithium carbonate treatment in depression. J Neuroscience and Mental Health, 2012,12(4):355-357

16. Celiac Disease. Rockville: Agency for Healthcare Research and Quality (US); 2004. [https://www.ncbi.nlm.nih.gov/books/NBK35149/].

17. Ji Shumei, Li Xiaojian, LiHuarong. Lithium carbonate combinating with paroxetine in treatment for patients with depression. Chinese Pharmacist,2008,11(11):1347-1349

18. Li Cheng and Xu Xiaofeng. Analysis of lithium carbonate in maintenance treatment for patients with depression. Occupationa and Health. 2001,17(4):123-124

19. Peng Dongying,Zuo Xiaoyun,Li Huarong. Fluoxetine combinating with lithium carbonate in treatment for patients with depression. Chinese Journal of New Drug and Clinic. 2007,26(4):289-291

20. Tan Bin.Clinical observation of sertaline and combination with lithium carbonate in treatmnet of patients with depression.Journal of Neuroscience and Mental health.2005,18(4):284-286

21. Wu Hongdong, Xiao Weibin, Zhang Weijuan.Cinical assement of lithium carbonate and combination with fluoxetine in prevention of switch to mania.Journal of Anti-infection Pharmacy,2015,12(6):934936,10.13493/j.issn.1672-7878.2015.06-052

22. Yuan Guofeng, Yu yuli,Zhang Yuqi. Blind assessemnt of low dose of lithium carbonate in maintenance treatment. Chinese Clinical Rehebilitation. 2005,9(8):50

23. Li Yongxi,Zhang Chunying. Comparison between cloimiprame and combination with lithium carbonate in treatment of patients with bipolar depression. Shandong Archieve of Psychiatry,2005,18(4)248-249

24. Ding Wantao. The lithium carbonate with imiprame in treatment for patients with depression. Annual meeting of Zhejiang province paychiatriy association, 2005.

25. Li Yongxi, Zhang Chunying. Yi Yanming. Comparson study of fluoxeting and combnation lithium carbonate in treatment for patients with depression. Sichuan Mental Health, 2006,19(3):172-173 
26. de Dios C, Goikolea JM, Colom F, Moreno C, Vieta E.Bipolar disorders in the new DSM-5 and ICD-11 classifications.Rev Psiquiatr Salud Ment. 2014;7(4):179-85. doi: 10.1016/j.rpsm.2014.07.005.

27. Debajyoti Bhowmik , Rajender R Aparasu, Suja S Rajan, Jeffrey T Sherer, Melissa Ochoa-Perez, Hua Chen.Risk of manic switch associated with antidepressant therapy in pediatric bipolar depression.J Child Adolesc Psychopharmacol. 2014 ;24(10):551-61. doi: 10.1089/cap.2014.0028.

28. Jin Weidong, Chen Jiong, Xing Baoping.An Investigation of clinical epidemilogy on switching associated with antidepressant.Part I:Prevanlence and its difference between different type of depression.2005,14(4):217-219,10.3969/j.issn.1005-0698.2005.04.008

29. Jin Weidonng,Wang Naixin, Wang Heqiu.The obsevation of switch induced by antidepressant in patients with deprssive patients. Chinese Journal of Psychiatry, 2009,42(2):128,10.3760/cma.j.issn.1006-7884.2009.02.022

30. Jin Weiding,Chen Zhengxin, Tong Zhenhua.Meta-Analysis for Incidence of Insomnia,Anxiety, Irritability and Manic Episode Induced by TCA and SSRIS. Herald of Medicine.2008,27(10):1190-1193,10.3870/j.issn.1004-0781.2008.10.019

31. Giacomo Salvadore, Jorge A Quiroz, Rodrigo Machado-Vieira, loline D Henter, Husseini K Manji, Carlos A Zarate Jr.The neurobiology of the switch process in bipolar disorder: a review.J Clin Psychiatry. 2010 ;71(11):1488-501. doi: 10.4088/JCP.09r05259gre.

32. Todor Dudev, Karine Mazmanian , Wei-Hsiang Weng , Cédric Grauffel , Carmay Lim .Free and Bound Therapeutic Lithium in Brain Signaling.Acc Chem Res. 2019, 15;52(10):2960-2970.doi: 10.1021/acs.accounts.9b00389.

\section{Figures}




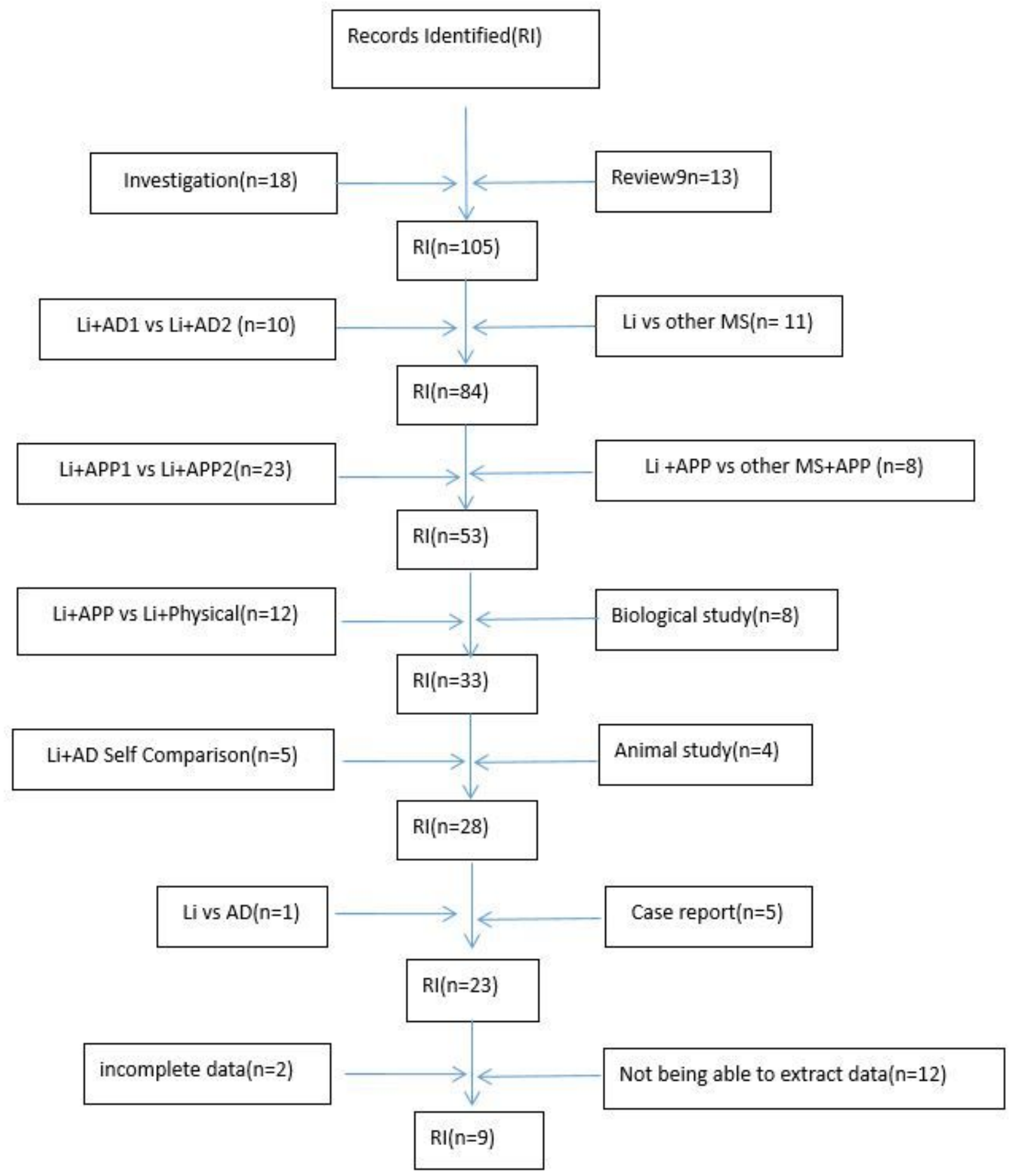

\section{Figure 1}

Search process $\mathrm{Li}=$ Lithium Carbonate; $\mathrm{AD}=$ Antidepressants; $\mathrm{APP}=$ Atypical antipsychotic; $\mathrm{MS}=$ Mood Stabilizer 
Experimental Control Odds Ratio Odds Ratio

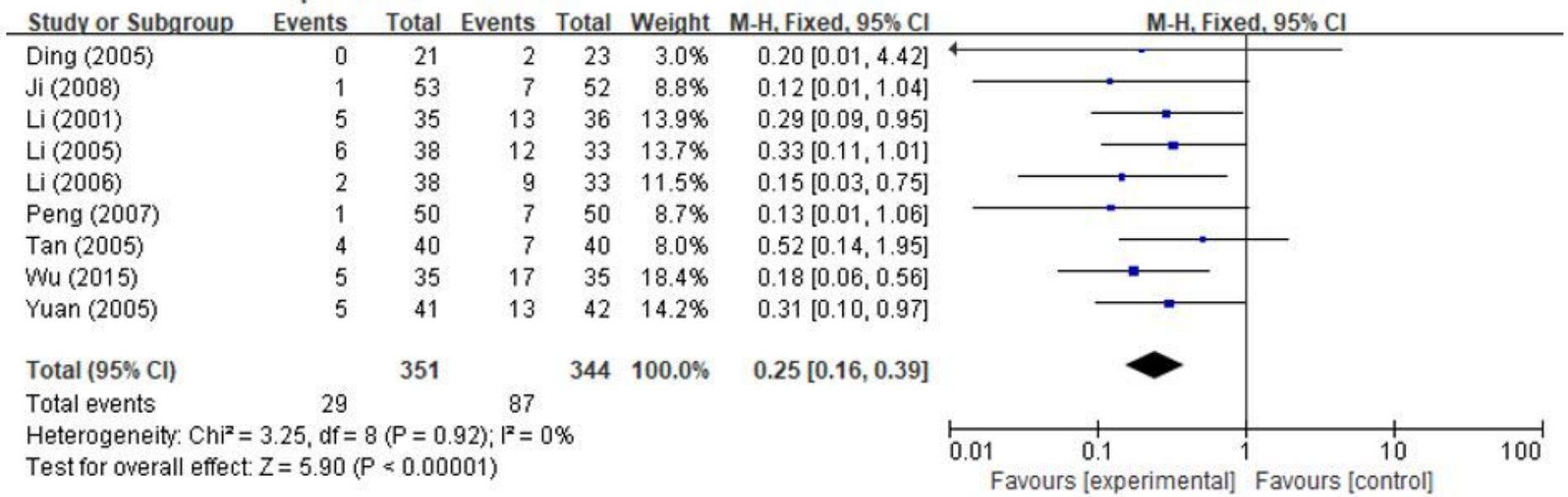

Figure 2

Comparison of switch rate between experimental group and control group.

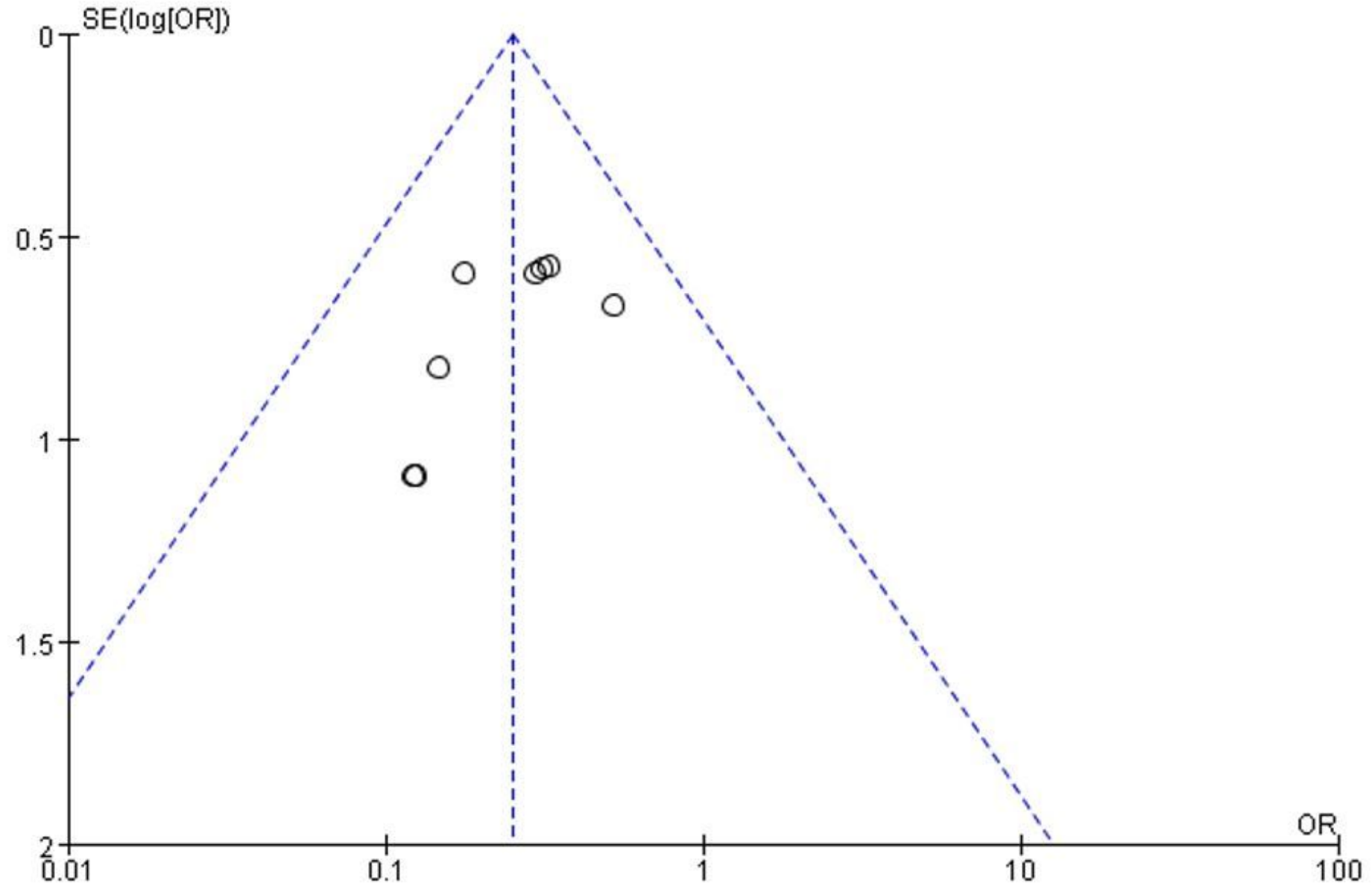

Cinurn?

Loading [MathJax]/jax/output/CommonHTML/fonts/TeX/fontdata.js 
The funnel plot analysis of study about switch rate

Egger's publication bias plot

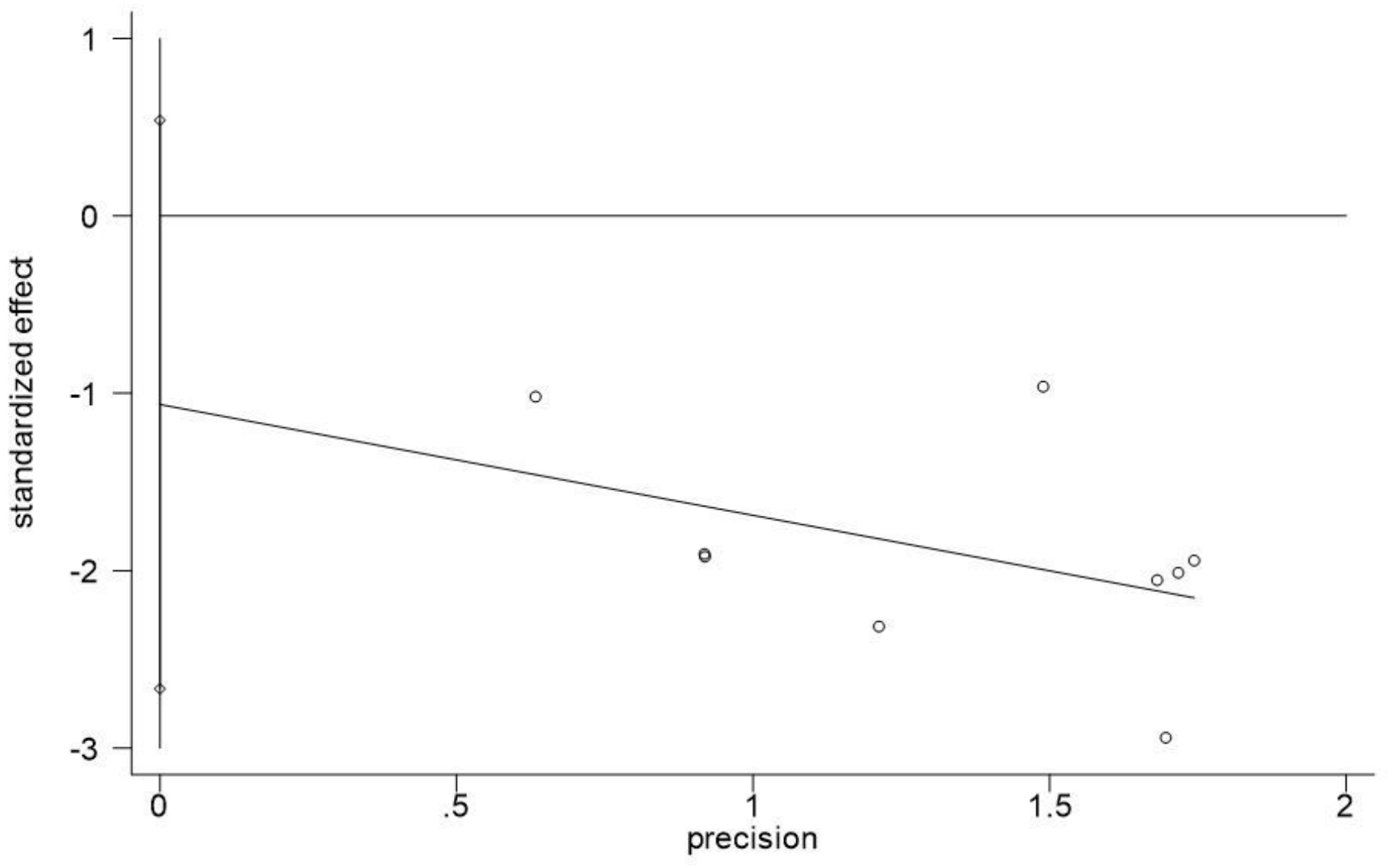

\section{Figure 4}

Egger`s publication bias test 


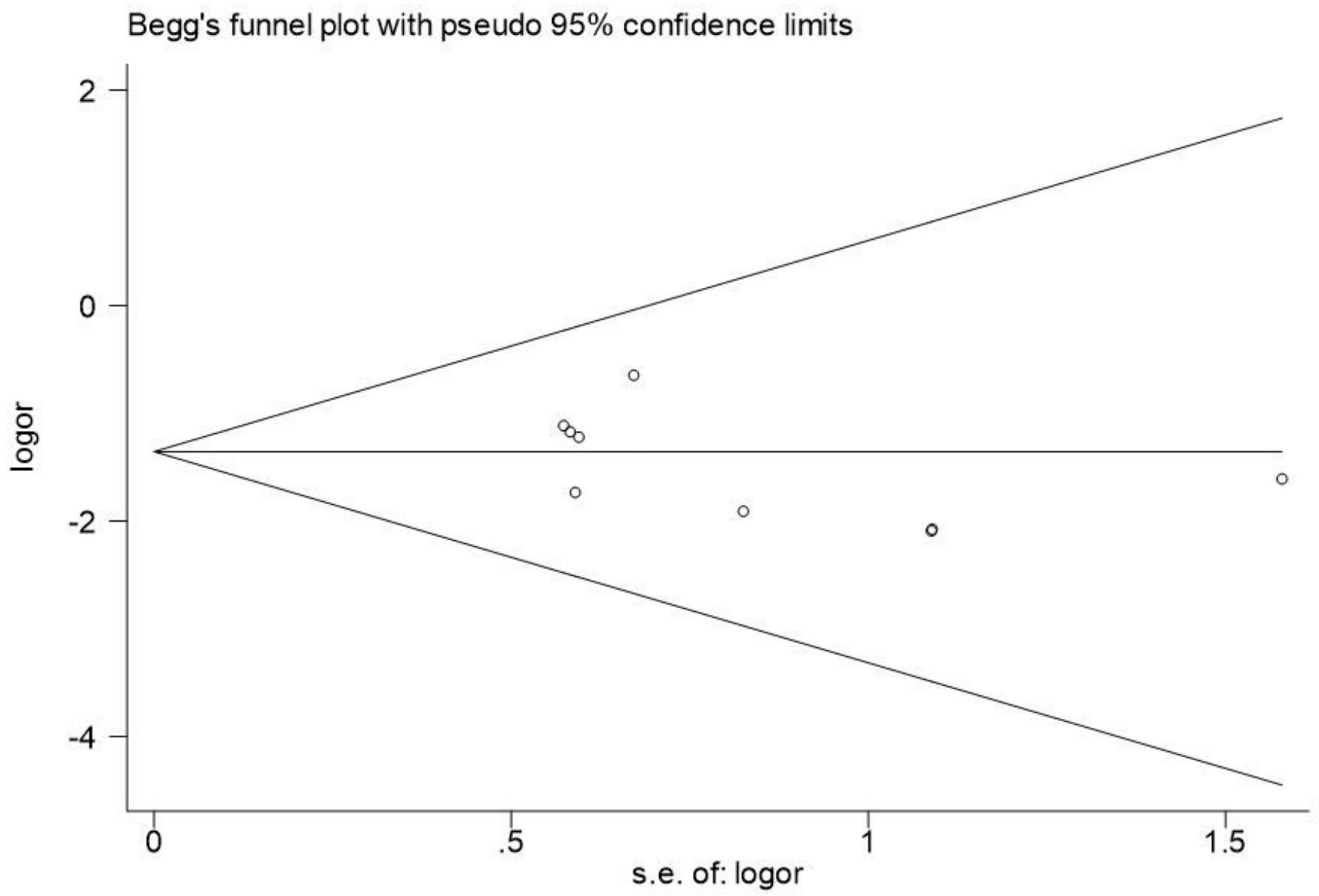

Figure 5

Begg`s publication bias test 
Experimental Control Odds Ratio Odds Ratio

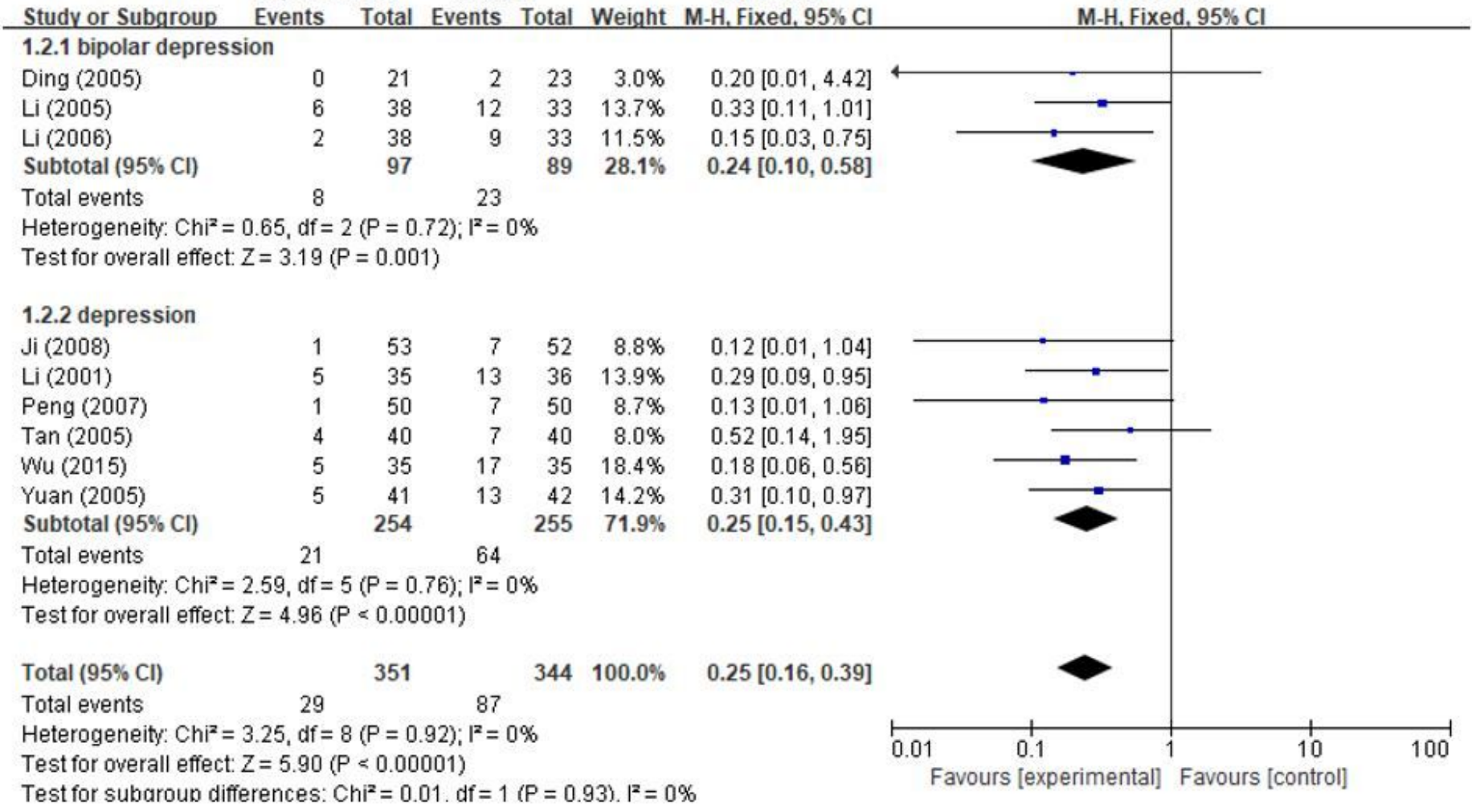

\section{Figure 6}

Subgroup comparison of switch rate according to type of depression. 


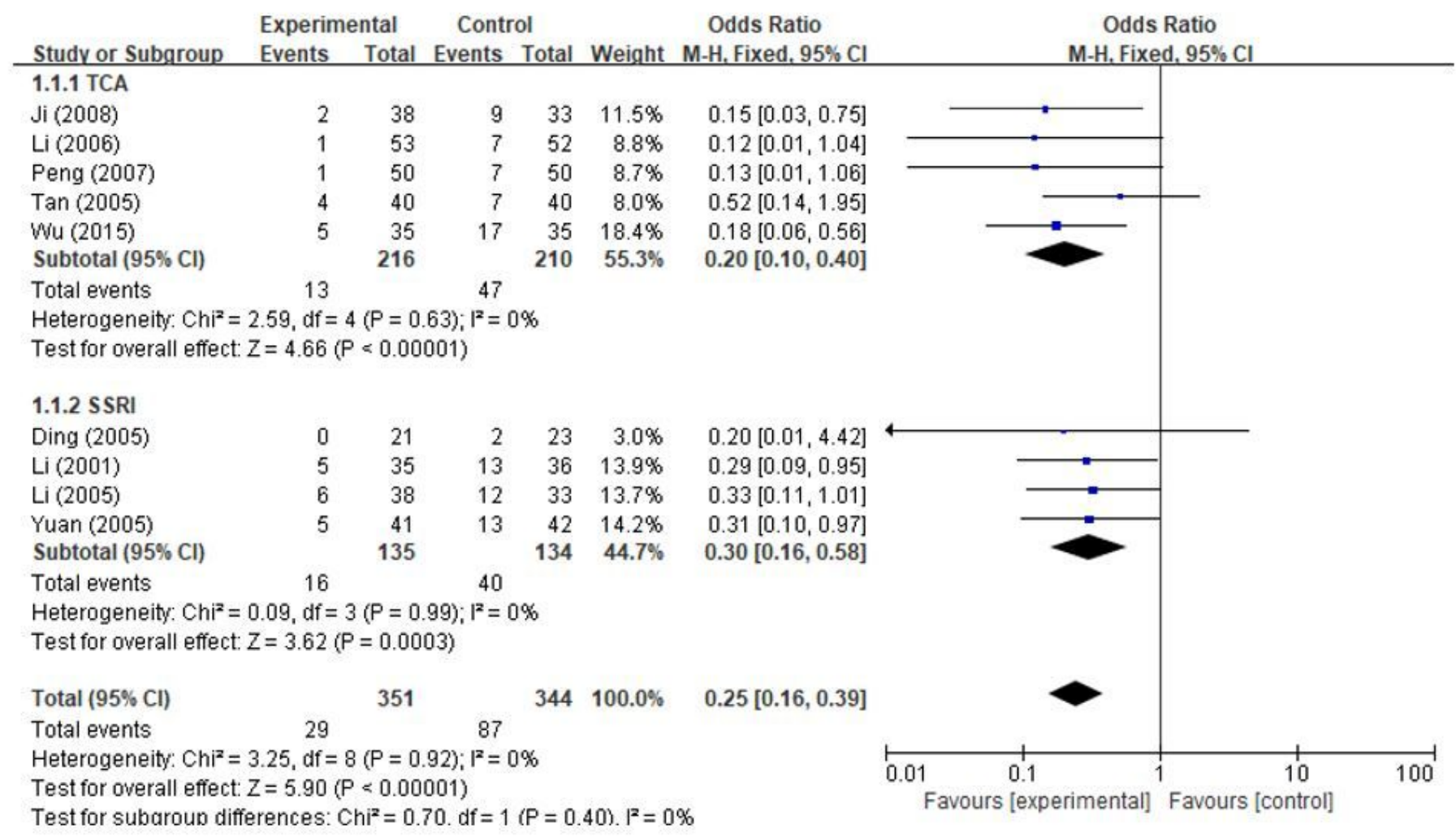

\section{Figure 7}

Subgroup comparison of switch rate according to type of antidepressant. 